\title{
Acute biliary events during anti- tuberculosis treatment: hospital case series and a nationwide cohort study
}

\author{
Lih-Yu Chang ${ }^{1}$, Chih-Hsin Lee ${ }^{2,3}$, Chia-Hao Chang ${ }^{1}$, Ming-Chia Lee ${ }^{4,5}$, Meng-Rui Lee', Jann-Yuan Wang ${ }^{6 *}$ (D) \\ and Li-Na Lee ${ }^{7}$
}

\begin{abstract}
Background: Tuberculosis (TB) remains one of the major infectious diseases worldwide. Adverse reactions are common during TB treatment. Few reports, however, are available on treatment-related acute biliary events (ABEs), such as cholelithiasis, biliary obstruction, acute cholecystitis, and cholangitis.

Methods: We first report four pulmonary TB patients who developed ABEs during anti-TB treatment. Abdominal sonography revealed multiple gall stones with dilated intrahepatic ducts in three patients and cholecystitis in one patient. To investigate the incidence of and risk factors for ABEs during anti-TB treatment, we subsequently conducted a nationwide cohort study using the National Health Insurance Research Database of Taiwan.

Results: A total of 159,566 pulmonary TB patients were identified from the database between 1996 and 2010, and among them, 195 (0.12\%) developed ABEs within 180 days after beginning anti-TB treatment. Logistic regression analysis revealed that the risk factors associated with ABEs are older age (relative risk [RR]: 1.32 [1.21-1.44] per 10year increment) and diabetes mellitus (RR: 1.59 [1.19-2.13]).

Conclusions: Although infrequently encountered, ABEs should be considered among patients with TB who experience abdominal discomfort with hyperbilirubinemia, especially patients who have older age or diabetes.
\end{abstract}

Keywords: Tuberculosis, Biliary event, Cholelithiasis, Cholecystitis, Cholangitis, National Health Insurance Research Database

\section{Background}

Tuberculosis (TB) remains one of the most deadly infectious diseases worldwide [1]. In 2015, approximately 10.4 million new cases of TB were diagnosed globally. Despite a decrease in the mortality rate in 2015 from 2000, TB caused approximately 1.8 million deaths in 2015 [1]. Although effective anti-TB drugs are readily available, the treatment of TB is not always successful because treatment-emergent adverse events-such as hepatotoxicity, peripheral neuropathy, gastrointestinal upset, hyperuricemia, optic neuritis, and cutaneous reactions [2] - often lead to treatment interruption and further dissemination of the TB bacilli.

\footnotetext{
* Correspondence: jywang@ntu.edu.tw

${ }^{6}$ Department of Internal Medicine, National Taiwan University Hospital, \#7, Chung-Shan South Road, Zhongzheng District, Taipei 10002, Taiwan Full list of author information is available at the end of the article
}

Hepatotoxicity is the most common adverse event associated with TB treatment, with an incidence rate between $10.2 \%$ and $18.9 \%$ in Taiwan, and it is potentially life-threatening [3-6]. The risk of drug-induced liver injury during anti-TB treatment ranges from 5\% to 33\% according to the American Thoracic Society [7]. Clinical presentations may include low-grade fever, general malaise, poor appetite, nausea, vomiting, abdominal distension, icteric conjunctiva, and elevated serum aminotransferases and bilirubin levels. These symptoms and signs are typically indistinguishable from those of acute biliary events (ABEs) such as cholelithiasis, biliary obstruction, acute cholecystitis, and cholangitis. Delayed or incorrect diagnosis of ABEs may cause unnecessary anti-TB treatment interruption, or if left untreated, may lead to intra-abdominal complications, such as acute pancreatitis, sepsis, or perforation [8]. However, the 
association between $\mathrm{ABEs}$ and anti-TB treatment has not been elucidated thus far. Therefore, in this paper, we first report four cases of ABEs during anti-TB treatment, and subsequently, we investigate the incidence rate and risk factors of $\mathrm{ABEs}$ during anti-TB treatment in a nationwide TB cohort.

\section{Methods}

This study consisted of two parts. In the first part (case series), we obtained the clinical presentations and treatment histories of patients with pulmonary TB who developed ABEs during anti-TB treatment at the National Taiwan University Hospital and its Hsinchu Branch between 2010 and 2015. In the second part (nationwide cohort), we conducted a nationwide cohort study using claims data from the National Health Insurance Research Database (NHIRD) of Taiwan to evaluate the incidence rate of $\mathrm{ABEs}$ during anti-TB treatment and identify risk factors for ABEs.

\section{Ethics statement}

The Institutional Review Board of the National Taiwan University Hospital and its Hsinchu Branch approved the study (NTUH REC: 201,309,064 W; NTUH-HC REC: 105-023-E). Due to the study's retrospective design, inform consent was deemed unnecessary.

\section{Identification of ABE cases in the hospital-based cohort (case series) \\ Definition of ABEs}

An adverse event was considered an $\mathrm{ABE}$ if it met both of the following criteria: (1) abnormal liver function during anti-TB treatment; and (2) diagnosis with image confirmation, such as abdominal computed tomography or sonography.

\section{Case selection}

Between 2010 and 2015, a total of 3686 patients with pulmonary TB were treated at the National Taiwan University Hospital $(n=2704)$ and its Hsinchu Branch $(n=982)$. Among them, 2879 (78.1\%) had cultureconfirmed pulmonary TB. Liver function (aspartate aminotransferase [AST], alanine aminotransferase [ALT], and total bilirubin) was evaluated at baseline and at least once during anti-TB treatment in 3675 (99.7\%) patients, and significant elevation in AST and ALT levels was noted in 416 (11.3\% of 3675$)$ patients. The medical records of these 416 patients were reviewed to determine whether $\mathrm{ABE}$ was responsible for abnormal liver function in these patients. The diagnosis of $\mathrm{ABE}$ was also confirmed using imaging modalities.

\section{Analysis of nationwide cohort Definition of ABEs}

An adverse event was considered an ABE if it met both of the following criteria: (1) diagnosis of cholelithiasis (ICD-9-CM code 574), acute cholecystitis (ICD-9-CM code 575.0-575.2), cholangitis (ICD-9-CM code 576.1), or obstruction of biliary tract (ICD-9-CM code 576.2); and (2) the patient had received at least one of the examinations and management therapies listed in Table 1. The primary outcome was ABEs that occurred within 180 days after commencement of anti-TB treatment.

\section{Case selection}

We identified patients in the NHIRD who had been diagnosed with pulmonary TB from 1996 to 2010 (Fig. 1). Active TB was defined by the following criteria: (1) at least two outpatient records or one inpatient record with a compatible diagnosis; (2) one prescription of at least three anti-TB medications; and (3) receiving two or more anti-TB medications simultaneously over a period of 120 days within a 180-day period [9]. Patients who were diagnosed with nontuberculous mycobacterial infection (ICD-9-CM code 031) during the last 60 days of anti-TB treatment were excluded. Patients who met any of the ABE diagnostic criteria before anti-TB treatment were also excluded. Date of treatment completion was defined as the last date on which two or more anti-TB drugs were taken simultaneously without receiving any anti-TB medication in the subsequent 60 days.

\section{Comorbidity and income status}

Comorbid conditions-comprising diabetes mellitus (DM), chronic obstructive pulmonary disease (COPD), malignancy, end-stage renal disease, connective tissue disease, acquired immunodeficiency syndrome, liver cirrhosis, transplantation, pneumoconiosis, and lowincome status-at the time TB diagnosis were identified by using previously published definitions $[9,10]$.

\section{Statistical analyses}

Intergroup differences were assessed by using independent-sample $t$ tests for continuous variables and chi-square or Fisher exact tests for categorical variables. The risk factors for ABEs in patients with pulmonary $\mathrm{TB}$ were evaluated using logistic regression analyses. Only variables with a two-sided $p<0.05$ were included in the final model. All analyses were performed using IBM SPSS Statistics Version 22 (IBM Corp., Armonk, NY, USA).

\section{Results}

Case series in the hospital cohort

Case 1

A male patient in his 80 s with comorbid, medically controlled hypertension and DM received standard four- 
Table 1 Examinations and management of acute biliary events

\begin{tabular}{|c|c|c|}
\hline & Examination or Management & NHIRD code \\
\hline \multirow[t]{3}{*}{ Non-invasive Study } & Cholescintigraphy & $26040 B$ \\
\hline & Oral cholecystography & $33020 B$ \\
\hline & Intravenous choledochocystography & $33021 \mathrm{~B}$ \\
\hline \multirow[t]{4}{*}{ Invasive Study } & Transduodenal choledochoscopy & $28032 B$ \\
\hline & Percutaneous transhepatic choledochoscopy & $28036 \mathrm{~B}$ \\
\hline & Endoscopic retrograde cholangiopancreatography & $33024 B$ \\
\hline & Percutaneous transhepatic cholangiography & $33025 B$ \\
\hline \multirow[t]{9}{*}{ Drainage } & Fiber choledochoscopy, percutaneous via T-tube or other tract & $28034 \mathrm{~B}$ \\
\hline & T-tube cholecystography & $33022 B$ \\
\hline & Percutaneous transhepatic cholangiography-drainage & $33026 \mathrm{~B}$ \\
\hline & Percutaneous gall bladder drainage & $33106 \mathrm{~B}$ \\
\hline & Endoscopic retrograde biliary drainage & $56020 B$ \\
\hline & Endoscopic nasobiliary drainage & $56021 \mathrm{~B}$ \\
\hline & Choledochoscopy and choledochotomy & $56034 \mathrm{~B}$ \\
\hline & Choledochotomy with T-tube drainage & $75208 B$ \\
\hline & Choledocholithotomy with T-tube drainage & $75209 B$ \\
\hline \multirow[t]{4}{*}{ Stone Removal } & Fiber choledochoscopy, percutaneous via T-tube or other tract, with removal of stones & 28008B \\
\hline & Fiber choledochoscopy, intraoperative, with removal of stones & $28035 B$ \\
\hline & PTCD-stone removal & 33083B \\
\hline & Choledocholithotomy (transduodenal) & $75202 B$ \\
\hline \multicolumn{3}{|l|}{ Operation } \\
\hline \multirow[t]{6}{*}{ Traditional } & Fiber choledochoscopy, intraoperative & 28007B \\
\hline & Operative cholangiography & 33023B \\
\hline & Cholecystostomy & $75201 B$ \\
\hline & Cholecystectomy & $75203 B$ \\
\hline & Choledochojejunostomy & 75204B \\
\hline & Cholecystoenterostomy & $75205 B$ \\
\hline \multirow[t]{2}{*}{ Laparoscopic } & Laparoscopic cholecystectomy & $75215 B$ \\
\hline & Laparoscopic choledocholithotripsy & $75218 B$ \\
\hline
\end{tabular}

drug anti-TB treatment (isoniazid, rifampin, ethambutol, and pyrazinamide) for his smear-positive, cultureconfirmed pulmonary TB. He experienced fever and chills after one week of treatment. Laboratory examinations showed elevated AST (212 IU/L) and ALT (375 IU/L) and direct-type hyperbilirubinemia (total bilirubin: $2.81 \mathrm{mg} / \mathrm{dL}$; direct bilirubin: $2.57 \mathrm{mg} / \mathrm{dL}$ ). Abdominal sonography revealed the presence of multiple gall stones and dilated intrahepatic ducts. Anti-TB treatment was discontinued and ceftazidime was prescribed. One month later, the patient underwent a laparoscopic cholecystectomy procedure, followed by the resumption of anti-TB treatment. Thereafter, ABEs were not noted.

\section{Case 2}

A male patient in his 50s with comorbid, medically controlled DM and COPD was diagnosed with pulmonary TB based on a histology report of a transbronchial lung biopsy and a mycobacterial culture of bronchial washing sample. The first 3 weeks of fourdrug anti-TB treatment were uneventful, but after 4 weeks of treatment, he experienced nausea and vomiting. Laboratory tests revealed elevated transaminase (AST: $144 \mathrm{IU} / \mathrm{L}$ and ALT: $358 \mathrm{IU} / \mathrm{L}$ ). A minimal amount of ascites in the right subdiaphragmatic area was observed using abdominal sonography. A small amount of ascites was also observed in the perigallbladder, right anterior pararenal, and pelvic space one week later using abdominal computed tomography. The patient was diagnosed with acute cholecystitis. Anti-TB treatment was interrupted and cefmetazole was administered. After his adverse reactions had resolved, anti-TB treatment was successfully resumed. 


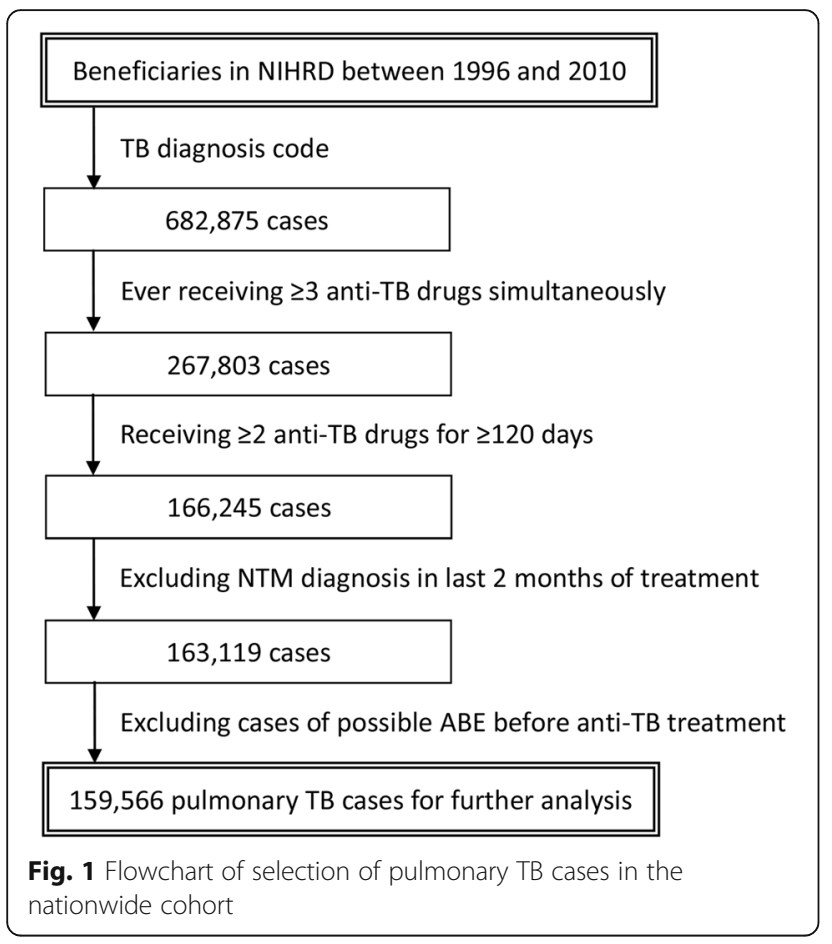

\section{Case 3}

A man in his 50s with comorbid hypertension and COPD, which were under irregular medical control, was diagnosed with smear-positive, culture-confirmed pulmonary TB. Two weeks prior to the commencement of standard anti-TB treatment, he had pneumonia with respiratory failure and septic shock. Laboratory tests demonstrated elevated transaminase levels, but normal total and direct bilirubin (AST: 144 IU/L; ALT: $358 \mathrm{IU} / \mathrm{L}$; and total bilirubin: $0.52 \mathrm{mg} / \mathrm{dL})$. Neither gallbladder stones nor bile duct stones were identified using computed tomography (slide thickness: $5 \mathrm{~mm}$; interval: $1.2 \mathrm{~mm}$ ). The patient was treated with courses of antibiotics, which improved his respiratory condition, hemodynamic condition, and aminotransferase levels (AST: $51 \mathrm{IU} / \mathrm{L}$; ALT: $23 \mathrm{IU} / \mathrm{L})$. The standard four-drug anti-TB treatment was started subsequently. However, after receiving anti-TB treatment for two weeks, his bilirubin level was increased, with a mild elevation in transaminase level (AST: 77 IU/L; ALT: $49 \mathrm{IU} / \mathrm{L}$; total bilirubin: $4.57 \mathrm{mg} /$ $\mathrm{dL}$; and direct bilirubin: $3.84 \mathrm{mg} / \mathrm{dL}$ ). Anti-TB treatment was halted. Multiple cholelithiasis with a dilated intrahepatic duct was diagnosed using abdominal sonography. Soon after the patient underwent the sonography procedure, he experienced another episode of pneumonia with septic shock and respiratory failure. Although bilirubin and transaminase both improved after interruption of anti-TB treatment for 10 days (AST: 61 IU/L; ALT: $38 \mathrm{IU} / \mathrm{L}$; total bilirubin: $1.72 \mathrm{mg} / \mathrm{dL}$; and direct bilirubin: $1.37 \mathrm{mg} / \mathrm{dL}$ ), the general condition of the patient deteriorated gradually. The patient died of refractory septic shock.

\section{Case 4}

A previously healthy male patient in his 20s was diagnosed with smear-positive, culture-confirmed pulmonary TB and TB pleurisy. No cholelithiasis was noted in abdominal sonographs obtained 2 years prior to the diagnosis of TB. The standard four-drug anti-TB treatment improved his TB clinical symptoms, including fever, chest pain, and left pleural effusion; however, abnormal liver function was noted (AST: $303 \mathrm{IU} / \mathrm{L}$; ALT: $499 \mathrm{IU} / \mathrm{L}$; and total bilirubin: $0.56 \mathrm{mg} / \mathrm{dL})$ after 6 weeks of treatment, which resolved (ASL: 26 IU/L; ALT: $34 \mathrm{IU} / \mathrm{L}$; total bilirubin: $0.1 \mathrm{mg} / \mathrm{dL}$ ) after the interruption of anti-TB treatment. In the 18th week, anti-TB treatment with isoniazid, rifampin, and ethambutol was resumed. However, the patient experienced acute abdominal cramping pain in the following week. His aminotransferase and bilirubin level did not increase (AST: 22 IU/L; ALT: 17 IU/L; and total bilirubin: $0.57 \mathrm{mg} / \mathrm{dL}$ ). Multiple small cholelithiases were discovered using abdominal sonography. Rechallenge with anti-TB treatment was complicated by recurrent abdominal cramping pain immediately after taking the anti-TB drugs. Because gastroenterologists suggested a possible diagnosis of intermittent biliary obstruction due to a passing stone, he received conservative management. The anti-TB treatment was successfully completed in the ninth month after TB diagnosis.

The demographic characteristics and clinical course of these four patients are summarized in Table 2 .

\section{NHIRD cohort}

From the beneficiaries listed in the NHIRD between 1996 and 2010, a total of 682,875 cases with diagnosis of pulmonary TB were identified (Fig. 1). A total of 159,566 cases that met the diagnostic criteria for pulmonary TB were included for further analysis. Subsequent analysis identified 195 (0.12\%) patients who developed ABEs within the initial 180 days of treatment. The patients who did not develop ABEs comprised the non-ABE group. The clinical characteristics of the $\mathrm{ABE}$ and nonABE groups-their age, sex, and underlying comorbidities-are summarized in Table 3. The mean age of the patients in the $\mathrm{ABE}$ and non-ABE groups was $66.2 \pm$ 13.5 years and $56.6 \pm 19.7$ years, respectively $(p<0.001)$. Men accounted for $67.7 \%$ of the ABE group and $68.6 \%$ of the non-ABE group $(p=0.075)$. Of the underlying comorbidities and income condition, a significant difference was only obtained between the $\mathrm{ABE}$ and non-ABE groups regarding DM prevalence $(p<0.001)$. The duration and type of anti-TB treatment were similar between the $\mathrm{ABE}$ and non-ABE groups, except for 
Table 2 Characteristics and clinical course of the cases of acute biliary events

\begin{tabular}{|c|c|c|c|c|}
\hline & Case 1 & Case 2 & Case 3 & Case 4 \\
\hline Age (decade of life) & 9th & 6th & 6th & $3 r d$ \\
\hline Sex & male & male & male & male \\
\hline Co-morbidity & DM & DM, COPD & HTN, COPD & Nil \\
\hline Anti-TB regimen before $A B E$ & HREZ & HREZ & HREZ & HRE \\
\hline Presenting symptoms & Fever/Chills & Nausea/Nomiting & Fever & Abdominal pain \\
\hline Peak ALT level $(U / L)^{a}$ & 375 & 303 & 16 & 499 \\
\hline Peak AST level $(U / L)^{b}$ & 212 & 223 & 98 & 303 \\
\hline Peak total bilirubin level $(\mathrm{mg} / \mathrm{dL})^{c}$ & 2.81 & 1.53 & 1.69 & 0.57 \\
\hline Diagnosis of $A B E$ & Cholecystitis & Cholecystitis & $\begin{array}{l}\text { Cholelithiasis with } \\
\text { biliary obstruction }\end{array}$ & $\begin{array}{l}\text { Cholelithiasis with } \\
\text { biliary obstruction }\end{array}$ \\
\hline Onset of $\mathrm{ABE}$ & 1 week later & 4 weeks later & 2 weeks later & 18 weeks later \\
\hline Treatment of $A B E$ & LC & Antibiotics & Antibiotics & Antibiotics \\
\hline
\end{tabular}

$A B E$ Acute biliary event, $A L T$ Alanine aminotransferase, AST Aspartate aminotransferase, COPD Chronic obstructive pulmonary disease, DM Diabetes mellitus, $E$ Ethambutol, $H$ Isoniazid, HTN Hypertension, $L C$ laparoscopic cholecystectomy, $R$ Rifampin, TB Tuberculosis, $Z$ Pyrazinamide

a normal range of ALT: $2-32 \mathrm{U} / \mathrm{L}$

${ }^{b}$ normal range of AST: $10-30 \mathrm{U} / \mathrm{L}$

cnormal range of total bilirubin: $0.2-1.2 \mathrm{mg} / \mathrm{dL}$

rifamycin prescription during the initial 60 and 180 days after TB diagnosis and isoniazid prescription during the initial 180 days.

The onset of ABEs was evenly distributed within the 180-day period (Fig. 2). The median number of days of onset was 76 days (IQR: 76-133) after initiation of antiTB treatment. Seventy-two (36.9\%) events occurred within the first 60 days of anti-TB treatment, 65 (33.3\%) within the next 60 days, and the remaining 58 (29.7\%) within the final 60 days. Among the 195 patients, 132 $(67.7 \%)$ received surgical intervention, which was either a traditional operation $(n=72)$ or laparoscopic surgery $(n=60)$ (Table 4). Twenty-four $(12.3 \%)$ received nonsurgical stone removal, and 66 (33.3\%) drainage.

Risk factors associated with ABEs were assessed using multivariable logistic regression analysis. We discovered that age $(p<0.001$; relative risk [RR]: 1.32 [1.21-1.44] per 10-year increment) and DM $(p<0.001 ; R R: 1.59$ [1.19-2.13]) were significantly associated with occurrence of ABEs.

\section{Discussion}

To our knowledge, this was the first study to investigate ABEs during anti-TB treatment. The incidence rate of ABEs during anti-TB treatment in the case study cohort was $0.11 \%(4 / 3686)$, which was similar to the finding in the nationwide cohort $(0.12 \%)$. ABEs do not appear to onset at a particular time. The risk factors for ABEs are older age and comorbid DM, with the latter occurring in half of the patients in the case series.

Because ethambutol and streptomycin are the only first-line TB therapies that are not associated with hepatotoxicity, abnormal liver function remains common during anti-TB treatment. Transient asymptomatic elevation of aminotransferase levels was observed in $10 \%$ to $20 \%$ of patients who have received isoniazid [11]. Furthermore, transient asymptomatic hyperbilirubinemia was detected in $0.6 \%$ of patients receiving rifampin [2]. The incidence of symptomatic and severe hepatotoxicity varies between different studies, ranging from 5\% to $33 \%$ [7]. However, the clinical presentations of hepatotoxicity are typically indistinguishable from those of ABEs. Distinguishing hepatotoxicity from ABEs has not been investigated in this field. Therefore, when a patient who is treated for $\mathrm{TB}$ presents symptoms of an $\mathrm{ABE}$-right upper quadrant pain, nausea, and vomiting with hyperbilirubinemia - the patient may be misdiagnosed with drug-induced hepatitis by the primary care physician. If the $\mathrm{ABE}$ is mild or self-limited, such as a passing stone, the physician may attribute the patient's recovery to discontinuation of anti-TB treatment, further delaying the diagnosis of an $\mathrm{ABE}$. Because some $\mathrm{ABE}$ cases should be managed aggressively with invasive procedures (more than two-thirds in the present nationwide cohort), delayed diagnosis may increase morbidity and mortality [12]. The findings of this study emphasize that although ABEs are not common, increasing awareness of these potential complications and practicing differential diagnosis are crucial for early diagnosis and proper management of ABEs.

From the nationwide cohort in this study, we discovered that older age and comorbid DM are two significant risk factors for ABEs during anti-TB treatment. These findings are similar to those of a previous multicenter study performed in Italy [13], which discovered that older age and DM are both 
Table $\mathbf{3}$ Characteristics of patients with and without acute biliary events

\begin{tabular}{|c|c|c|}
\hline & ABE group $(n=195)$ & Non-ABE group $(n=159,371)$ \\
\hline Age (years) $)^{a^{*}}$ & $66.2 \pm 13.5$ & $56.6 \pm 19.7$ \\
\hline Male & $132(67.7)$ & 109,399 (68.6) \\
\hline \multicolumn{3}{|l|}{ Tuberculosis diagnostic year ${ }^{\mathrm{b}}$} \\
\hline $1996-2000$ & $60(30.8)$ & $48,844(30.6)$ \\
\hline $2001-2005$ & $73(37.4)$ & $59,424(37.3)$ \\
\hline $2006-2010$ & $62(31.8)$ & $51,103(32.1)$ \\
\hline \multicolumn{3}{|l|}{ Co-morbidity (\%) } \\
\hline Diabetes mellitus $\mathrm{b}^{\mathrm{w}^{*}}$ & $63(37.4)$ & $38,075(23.9)$ \\
\hline Chronic obstructive pulmonary disease ${ }^{\mathrm{b}}$ & $10(5.1)$ & $7918(5.0)$ \\
\hline Malignancy ${ }^{b}$ & $12(6.2)$ & $6712(4.2)$ \\
\hline End-stage renal disease ${ }^{c}$ & $2(1.0)$ & $1998(1.3)$ \\
\hline Connective tissue disease $^{c}$ & $0(0.0)$ & $1151(0.7)$ \\
\hline Acquired immunodeficiency syndrome ${ }^{c}$ & $1(0.5)$ & $562(0.4)$ \\
\hline Liver cirrhosis $^{c}$ & $1(0.5)$ & $323(0.2)$ \\
\hline Transplantation $^{c^{*}}$ & $1(0.5)$ & $137(0.1)$ \\
\hline Pneumoconiosis $^{c}$ & $0(0)$ & $85(0.1)$ \\
\hline Low income $e^{b}$ & $5(2.6)$ & $4830(3.0)$ \\
\hline Total duration of anti-TB treatment & $266.9 \pm 99.7$ & $260.4 \pm 105.8$ \\
\hline \multicolumn{3}{|l|}{ Intensive phase (initial 60 days) ${ }^{a}$} \\
\hline No. of days covered by isoniazid ${ }^{a}$ & $47.7 \pm 18.6$ & $50.0 \pm 11.2$ \\
\hline No. of days covered by rifamycin ${ }^{a^{*}}$ & $48.9 \pm 13.4$ & $51.7 \pm 11.2$ \\
\hline No. of days covered by ethambutol ${ }^{a}$ & $51.2 \pm 11.4$ & $50.8 \pm 13.1$ \\
\hline No. of days covered by pyrazinamide ${ }^{a}$ & $39.9 \pm 21.3$ & $42.5 \pm 20.4$ \\
\hline \multicolumn{3}{|l|}{ Initial 180 days } \\
\hline No. of days covered by isoniazid ${ }^{\mathrm{a}^{*}}$ & $134.4 \pm 52.2$ & $142.4 \pm 53.4$ \\
\hline No. of days covered by rifamycin ${ }^{a^{*}}$ & $140.1 \pm 37.9$ & $152.5 \pm 32.8$ \\
\hline No. of days covered by ethambutol ${ }^{a}$ & $145.2 \pm 35.6$ & $141.9 \pm 42.2$ \\
\hline No. of days covered by pyrazinamide ${ }^{a}$ & $67.8 \pm 44.9$ & $69.3 \pm 45.8$ \\
\hline
\end{tabular}

Data are presented as number (\%) or mean \pm standard deviation

${ }^{a}$ Compared using an independent-sample $t$ test

${ }^{\mathrm{b}}$ Compared using a chi-square test

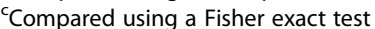

${ }^{*} p<0.05$ for the comparison between the ABE and non-ABE groups

independent risk factors for cholelithiasis. Epidemiological studies on the risk of gallbladder disease among patients with DM have yielded inconsistent results. In a case-control study [14], the prevalence of DM was significantly higher in patients with gallstone than in the control group $(11.6 \%$ vs. $4.8 \%$, odds ratio 2.55 [1.39-4.67]). In studies demonstrating a positive association between DM and gallbladder disease or gallstones, the risk ratios have been reported to lie in the range 1.68-2.09 [15-18]. Other studies, however, have not found an association $[19,20]$. The results of a recent meta-analysis suggested that a diagnosis of DM may increase the relative risk of gallbladder disease by $56 \%$ [21].
Patients with ABEs during anti-TB treatment may have asymptomatic cholelithiasis before treatment. When treatment begins, isoniazid and rifampin may inhibit the bile salt export pump [22] and accelerate the formation of cholesterol stone [23], with the previous asymptomatic stone used as a nucleus, resulting in a higher risk of ABEs. Cholelithiasis may be caused by either cholesterol gallstones, which are a mixture of cholesterol, bile salt, and phospholipids, or pigmented gallstones. The relative proportion of each component predicts if the mixture of cholesterol, bile salt, and phospholipids forms crystals, micelles, or vesicle micelles [24]. Crystal formation is associated with higher proportion of bile salt. The crystal becomes the nucleus of a cholesterol gallstone. Insulin 


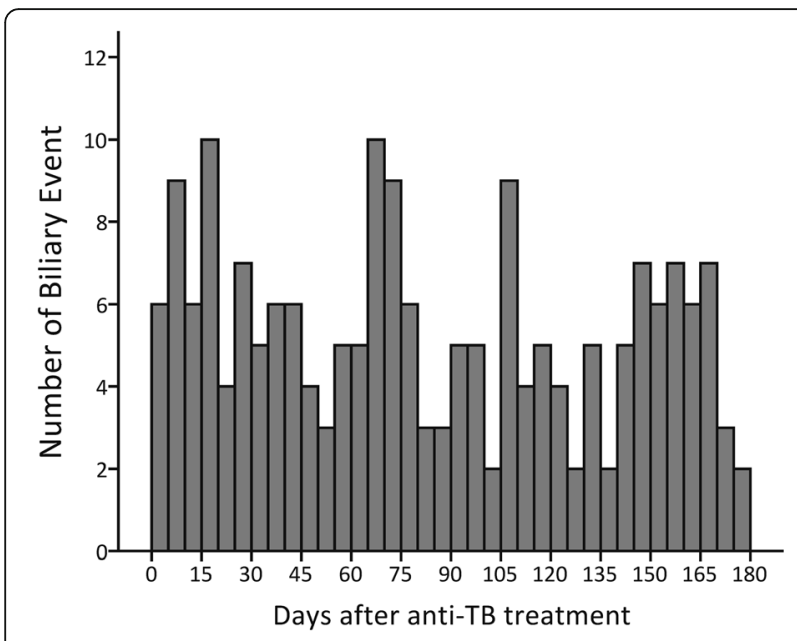

Fig. 2 Time of onset of acute biliary events

treatment has been shown to increase the biliary saturation index, resulting in cholesterol precipitation and gallstone formation [25, 26].

Another possible mechanism for the increased risk of an $\mathrm{ABE}$ in patients with $\mathrm{DM}$ may be decreased gallbladder motility as a result of denervation [27, 28]. The underlying pathogenesis is still unclear. One possibility is fewer cholecystokinin receptors on the gallbladder wall due to diabetic autonomic neuropathy leading to poor response to cholecystokinin stimulation [27]. In addition to impaired cholinergic innervation, cholecystoparesis may result from increased dopaminergic activity [29-32].

We discovered that duration of rifamycin use in the $A B E$ group was significantly shorter than that in the non$\mathrm{ABE}$ group. This finding is most likely to be confounded by indication. Among the first-line anti-TB drugs, only rifampin has been reported to be associated with cholestasis, which typically occurs in the first month of treatment [7]. Rifampin inhibits the major bile salt exporter pump and blocks bilirubin uptake [33]. In addition, rifampin competitively affects the clearance of bilirubin from the sinusoidal membrane or canaliculular level in a dose-

Table 4 Management and intervention for acute biliary events in the nationwide cohort

\begin{tabular}{ll}
\hline & $\begin{array}{l}\text { ABE Group } \\
(\mathrm{n}=195)\end{array}$ \\
\hline Non-invasive study (excluding abdominal sonography) & $5(2.6 \%)$ \\
Invasive study & $63(33.3 \%)$ \\
Drainage & $66(33.8 \%)$ \\
Stone removal & $24(12.3 \%)$ \\
Operation & $132(67.7 \%)$ \\
$\quad$ Traditional operation & $72(36.9 \%)$ \\
$\quad$ Laparoscopic operation & $60(30.8 \%)$ \\
\hline
\end{tabular}

dependent manner [34, 35]. Therefore, when the bilirubin level increases, primary care physicians tend to discontinue rifamycin treatment first. However, it was impossible to confirm the effect of rifampin on the development of ABEs in this retrospective study. Because it is unethical to treat $\mathrm{TB}$ without using rifamycin, a prospective interventional study comparing different doses of rifampin could be used to address this issue.

The present study had some limitations. First, because of the retrospective design of the study, the incidence of ABEs was probably underestimated in the hospital-based cohort because of missing mild and transient events. However, the effect of this underestimation may not be large because serum transaminase and bilirubin assessments in the second, fourth, and eighth weeks of treatment are recommended by $\mathrm{TB}$ treatment guidelines in Taiwan [36], and the percentage of follow-up tests performed for liver function in the hospital-based cohort was $>90 \%$. Second, because the NHIRD cohort study was based solely on claims data, $\mathrm{ABE}$ incidence may have been underestimated because of noncomprehensive data. Finally, the causal relationships between ABEs, rifampin treatment, and comorbid DM were not addressed. Further prospective studies are necessary to clarify these issues.

\section{Conclusion}

Although uncommon, ABEs develop in approximately $0.12 \%$ of patients with pulmonary $\mathrm{TB}$ receiving anti-TB treatment. Clinicians should be aware of this potential complication of anti-TB treatment and maintain high clinical suspicion for patients with pulmonary $\mathrm{TB}$ who experience abdominal discomfort during anti-TB treatment, especially elderly patients and those with DM.

\section{Abbreviations}

ABE: Acute biliary event; ALT: Alanine aminotransferase; AST: Aspartate aminotransferase; COPD: Chronic obstructive pulmonary disease;

DM: Diabetes mellitus; NHIRD: National Health Insurance Research Database; RR: Relative risk; TB: Tuberculosis

\section{Acknowledgements}

The authors thank the National Health Research Institute of Taiwan for providing access to the National Health Insurance Research Database.

\section{Funding}

This study was funded by the Taiwan Ministry of Science and Technology (NSC101-3114-Y-002-003, MOST104-2321-B-002-058) and the Ministry of Health and Welfare (MOHW105-CDC-C-114-00103). The funders had no role in the study design, data collection and analysis, decision to publish, or preparation of the manuscript. Guarantor of the paper JYW.

\section{Availability of data and materials}

Details of the computer code for statistical analyses are available from the corresponding author at: jywang@ntu.edu.tw.

\section{Authors' contributions}

LYC drafted the manuscript and designed the study with JYW and CHL. CHL and $M C L$ were involved in data processing. JYW, LYC, CHC, and MRL performed statistical analysis. LNL was the director responsible for general 
organization and instruction. All authors reviewed, provided input and approved the final manuscript.

\section{Ethics approval and consent to participate}

The Institutional Review Board of the National Taiwan University Hospital and its Hsinchu Branch approved the study (NTUH REC: 201,309,064 W; NTUH-HC REC: 105-023-E). Due to the retrospective design, inform consent was deemed unnecessary.

\section{Consent for publication}

Written informed consents of the four illustrated cases for publication were obtained.

\section{Competing interests}

The authors declare that they have no competing interests.

\section{Publisher's Note}

Springer Nature remains neutral with regard to jurisdictional claims in published maps and institutional affiliations.

\section{Author details}

'Department of Internal Medicine, National Taiwan University Hospital, Hsinchu Branch, Hsinchu, Taiwan. 'Division of Pulmonary Medicine, Wanfang Hospital, Taipei Medical University, Taipei, Taiwan. ${ }^{3}$ School of Medicine, College of Medicine, Taipei Medical University, Taipei, Taiwan. ${ }^{4}$ Department of Pharmacy, New Taipei City Hospital, New Taipei City, Taiwan. ${ }^{5}$ School of Pharmacy, College of Pharmacy, Taipei Medical University, Taipei, Taiwan. ${ }^{6}$ Department of Internal Medicine, National Taiwan University Hospital, \#7, Chung-Shan South Road, Zhongzheng District, Taipei 10002, Taiwan. 'Department of Laboratory Medicine, National Taiwan University Hospital, Taipei, Taiwan

Received: 4 July 2017 Accepted: 17 January 2018

\section{Published online: 01 February 2018}

\section{References}

1. WHO: Global tuberculosis report 2016. In. Genva, Switzland: WHO Press; 2016.

2. American Thoracic S, CDC, Infectious Diseases Society of American: Treatment of tuberculosis. MMWR Recomm Rep 2003, 52(RR-11):1-77.

3. Huang YS, Chern HD, Su WJ, Wu JC, Lai SL, Yang SY, et al. Polymorphism of the N-acetyltransferase 2 gene as a susceptibility risk factor for antituberculosis drug-induced hepatitis. Hepatology. 2002;35(4):883-9.

4. Wang JY, Liu CH, Hu FC, Chang HC, Liu JL, Chen JM, et al. Risk factors of hepatitis during anti-tuberculous treatment and implications of hepatitis virus load. J Inf Secur. 2011;62(6):448-55.

5. Wang JY, Lee LN, Yu CJ, Chien YJ, Yang PC, Tami G. Factors influencing time to smear conversion in patients with smear-positive pulmonary tuberculosis. Respirology. 2009:14(7):1012-9.

6. Shu CC, Lee CH, Lee MC, Wang JY, Yu CJ, Lee LN. Hepatotoxicity due to first-line anti-tuberculosis drugs: a five-year experience in a Taiwan medical centre. Int J Tuberc Lung Dis. 2013;17(7):934-9.

7. Saukkonen JJ, Cohn DL, Jasmer RM, Schenker S, Jereb JA, Nolan CM, et al. An official ATS statement: hepatotoxicity of antituberculosis therapy. Am J Respir Crit Care Med. 2006;174(8):935-52.

8. Schmidt M, Sondenaa K, Vetrhus M, Berhane T, Eide GE. Long-term followup of a randomized controlled trial of observation versus surgery for acute cholecystitis: non-operative management is an option in some patients. Scand J Gastroenterol. 2011;46(10):1257-62.

9. Wang JY, Lee MC, Shu CC, Lee CH, Lee LN, Chao KM, et al. Optimal duration of anti-TB treatment in patients with diabetes: nine or six months? Chest. 2015;147(2):520-8.

10. Wang JY, Sun HY, Wang JT, Hung CC, Yu MC, Lee CH, et al. Nine- to twelvemonth anti-tuberculosis treatment is associated with a lower recurrence rate than 6-9-month treatment in human immunodeficiency virus-infected patients: a retrospective population-based cohort study in Taiwan. PLoS One. 2015:10(12):e0144136.

11. Mitchell JR, Zimmerman HJ, Ishak KG, Thorgeirsson UP, Timbrell JA, Snodgrass WR, et al. Isoniazid liver injury: clinical spectrum, pathology, and probable pathogenesis. Ann Intern Med. 1976;84(2):181-92.
12. Kimura Y, Takada T, Strasberg SM, Pitt HA, Gouma DJ, Garden OJ, et al. TG13 current terminology, etiology, and epidemiology of acute cholangitis and cholecystitis. J Hepatobiliary Pancreat Sci. 2013;20(1):8-23.

13. Attili AF, Capocaccia R, Carulli N, Festi D, Roda E, Barbara L, et al. Factors associated with gallstone disease in the MICOL experience. Multicenter Italian study on epidemiology of Cholelithiasis. Hepatology. 1997;26(4):809-18.

14. De Santis A, Attili AF, Ginanni Corradini S, Scafato E, Cantagalli A, De Luca C, et al. Gallstones and diabetes: a case-control study in a free-living population sample. Hepatology. 1997;25(4):787-90.

15. Campbell PT, Newton CC, Patel AV, Jacobs EJ, Gapstur SM. Diabetes and cause-specific mortality in a prospective cohort of one million U.S. adults. Diabetes Care. 2012;35(9):1835-44.

16. Chen JY, Hsu CT, Liu JH, Tung TH. Clinical predictors of incident gallstone disease in a Chinese population in Taipei, Taiwan. BMC Gastroenterol. 2014;14:83.

17. Festi D, Dormi A, Capodicasa S, Staniscia T, Attili AF, Loria P, et al. Incidence of gallstone disease in Italy: results from a multicenter, population-based Italian study (the MICOL project). World J Gastroenterol. 2008;14(34):5282-9.

18. Noel RA, Braun DK, Patterson RE, Bloomgren GL. Increased risk of acute pancreatitis and biliary disease observed in patients with type 2 diabetes: a retrospective cohort study. Diabetes Care. 2009;32(5):834-8.

19. Boland LL, Folsom AR, Rosamond WD. Atherosclerosis risk in communities study I: Hyperinsulinemia, dyslipidemia, and obesity as risk factors for hospitalized gallbladder disease. A prospective study. Ann Epidemiol. 2002; 12(2):131-40.

20. Halldestam I, Kullman E, Borch K. Incidence of and potential risk factors for gallstone disease in a general population sample. Br J Surg. 2009:96(11): 1315-22.

21. Aune $D$, Vatten $\sqcup$. Diabetes mellitus and the risk of gallbladder disease: a systematic review and meta-analysis of prospective studies. J Diabetes Complicat. 2016 Mar;30(2):368-73.

22. Gou XY, Xu XF, Zhang QZ, Li C, Deng Y, Jiang Y, et al. The inhibition of hepatic bile acids transporters Ntcp and Bsep is involved in the pathogenesis of isoniazid/rifampicin-induced hepatotoxicity. Toxicol Mech Methods. 2015;25(5):382-7

23. Kong FM, Sui CY, Li YG, Guo KJ, Guo RX. Hepatobiliary membrane transporters involving in the formation of cholesterol calculus. Hepatobiliary Pancreat Dis Int. 2006 May:5(2):286-9.

24. Van Erpecum KJ. Pathogenesis of cholesterol and pigment gallstones: an update. Clin Res Hepatol Gastroenterol. 2011:35(4):281-7.

25. Bennion LJ, Grundy SM. Effects of diabetes mellitus on cholesterol metabolism in man. N Engl J Med. 1977;296(24):1365-71.

26. Ponz De Leon M, Ferenderes $\mathrm{R}$, Carulli N. Bile composition in patients with high risk of cholelithiasis. Minerva Med. 1976;67(53):3483-90.

27. Pazzi P, Scagliarini R, Gamberini S, Pezzoli A. Review article: gall-bladder motor function in diabetes mellitus. Aliment Pharmacol Ther. 2000;14(Suppl 2):62-5.

28. Shaw SJ, Hajnal F, Lebovitz Y, Ralls P, Bauer M, Valenzuela J, et al. Gallbladder dysfunction in diabetes mellitus. Dig Dis Sci. 1993;38(3):490-6.

29. Catnach SM, Ballinger AB, Stevens M, Fairclough PD, Trembath RC, Drury PL, et al. Erythromycin induces supranormal gall bladder contraction in diabetic autonomic neuropathy. Gut. 1993;34(8):1123-7.

30. Braverman DZ. The lack of effect of metoclopramide on gallbladder volume and contraction in diabetic cholecystoparesis. Am J Gastroenterol. 1986:81(10):960-2.

31. Mansi C, Savarino V, Vigneri S, Sciaba L, Perilli D, Mele MR, et al. Effect of D2 dopamine receptor antagonist levosulpiride on diabetic cholecystoparesis: a double-blind crossover study. Aliment Pharmacol Ther. 1995:9(2):185-9.

32. Kapicioglu S, Senturk O, Bambul N, Ilgun K. Action of cisapride on gallbladder contraction in patients with diabetes mellitus. HepatoGastroenterology. 1998;45(23):1410-4.

33. Byrne JA, Strautnieks SS, Mieli-Vergani G, Higgins CF, Linton KJ, Thompson RJ. The human bile salt export pump: characterization of substrate specificity and identification of inhibitors. Gastroenterology. 2002;123(5):1649-58.

34. Chitturi S, Farrell GC. Drug-induced liver disease. Curr Treat Options Gastroenterol. 2000;3(6):457-62

35. Capelle P, Dhumeaux D, Mora M, Feldmann G, Berthelot P. Effect of rifampicin on liver function in man. Gut. 1972;13(5):366-71.

36. TSTLD. Taiwan guidelines for TB diagnosis and treatment. 5.1th ed. Taiwan: TSTLD Press; 2015 